\title{
AJCC v6 Stage
}

National Cancer Institute

\section{Source}

National Cancer Institute. A/CC v6 Stage. NCI Thesaurus. Code C90529.

A cancer stage defined according to the AJCC 6th edition criteria. 